\title{
GFEM AND LSFEM IN THE SOLUTION OF THE TRANSIENT BIDIMENSIONAL CONVECTION-DIFFUSION EQUATION
}

\author{
E. C. Romão ${ }^{\mathrm{a}}$, \\ J. B. Aparecido ${ }^{\text {, }}$ \\ J. B. Campos-Silvab, \\ and L. F. M. de Moura ${ }^{a}$ \\ ${ }^{a}$ Universidade Estadual de Campinas \\ Faculdade de Engenharia Mecânica \\ Departamento de Engenharia Térmica e \\ Fluidos \\ Rua Mendeleyev, 200 \\ Cidade Universitária "Zeferino Vaz" \\ Distrito de Barão Geraldo \\ CEP:13083-860 Campinas SP Brasil \\ estaner23@yahoo.com.br \\ buniversidade Estadual Paulista \\ Departamento de Engenharia Mecânica \\ Av. Brasil Centro, 56 \\ CEP - 15385-000 - Ilha Solteira SP Brasil
}

\begin{abstract}
Convection dominated flows result in a hyperbolic system of equations which leads to ill-conditioned matrices and oscillatory approximations when using the classical Galerkin Finite Element Method (GFEM). In this paper, the Least Square Finite Method (LSFEM) is introduced in the study of transient bidimensional convection-diffusion problems. The differentiated equation of second order which describes the convective-diffusive phenomenon is transformed into an equivalent system of partial differentiated equations of first order which is discretized by the formulation of the LSFEM resulting in a defined algebraic, symmetrical and positive system. The performance of the method is verified by the solution of a test- problem.
\end{abstract}

Keywords: Finite Element Method, Least Squares, Galerkin, ConvectionDiffusion, Transients Problems.

\section{NOMENCLATURE}

$k \quad$ thermal conductivity

$x, y \quad$ space coordinates

$t$ time coordinate

$f$ source

$N_{j} \quad$ interpolation function

$N_{\text {node }}$ number of nodes in each finite element

$q_{x} \quad$ heat flux along the x-axis

$q_{y} \quad$ heat flux along the y-axis

$\hat{q}_{x}^{e} \quad$ heat flux approximation along x-axis

$\hat{q}_{y}^{e} \quad$ heat flux approximation along t-axis

$u$ temperature

$\hat{u}_{j}^{e} \quad$ temperature approximation in the finite element

$v \quad$ weight function

$v_{i}^{e} \quad$ weight function in the element

$R_{j} \quad$ residual equations

$\mathfrak{R}$ real numbers

\section{Greek symbols}

$\Omega \quad$ two-dimensional domain

$\Omega^{e} \quad$ two-dimensional domain in the element

$\alpha \quad$ coefficient of Crank-Nicholson Method

\section{Subscripts}

node identification of a node

\section{INTRODUCTION}

From the 50s on, some researchers such as Turner et al. (1956), Clough (1960) and Argyris (1963) started to use the Least Square Finite Element Method in its patterned format and the Galerkin Method to solve dominant diffusive problems and they had excellent results.

However most part of the Mechanics of Fluids problems and Heat and Mass Transfer are classified as dominant convective. Several authors presented papers in the last decades demonstrating that the application of the Galerkin Method in the solution of dominant convective problems in transient regime generate numerical oscillations, for example, Camprub et al. (2000), Romão (2004) e Romão et al. (2008), fact that could be avoided only with a large refinement of the mesh what would be computationally and financially expensive for bi and three-dimensional problems.

In this paper, the application of the Least Square Finite Method in the numerical solution of dominant convective phenomenon in transient regimes on a bidimensional domain will be presented. The application of the LSFEM always yield to an algebraic system of equations symmetrical and 
positive defined that can be solved, for example, by conjugate gradient methods and the solutions are free of oscillations, even in problems with the convective phenomenon dominant.

Firstly, the time discretization of the partial differential equations which model the transient convective-diffusive phenomenon will be presented followed by the spatial discretization. For the time discretization, the Crank-Nicolson scheme has been used for the spatial discretization, the Galerkin Finite Element Method (GFEM) and the Least Square Finite Element Method (LSFEM) are applied, in order to compare the solutions.

\section{EQUATION MODEL}

It is introduced here a numerical study of the partial differential equation that models a generical transient bidimensional convective-diffusive phenomenon defined in the $\Theta=\Xi \otimes \Omega \subset \mathfrak{R}^{3}$ domain in which $\Xi$ and $\Omega$ are closed and bounded domains. The governing equation is:

$$
\begin{gathered}
\psi \frac{\partial u}{\partial t}+\frac{\partial}{\partial x}\left(k \frac{\partial u}{\partial x}\right)+\frac{\partial}{\partial y}\left(k \frac{\partial u}{\partial y}\right)+A_{x} \frac{\partial u}{\partial x}+ \\
A_{y} \frac{\partial u}{\partial y}+B u+f=0
\end{gathered}
$$

in which is admitted $k=$ constant $\neq 0, u=u(x, y)$, $A_{x}=A_{x}(x, y), A_{y}=A_{y}(x, y), B=B(x, y), \Psi=\Psi(x, y)$ and $f=f(x, y)$ with $x, y, t \in \mathfrak{R}$ with boundary conditions of the first and second type as well the initial condition.

\section{TIME DISCRETIZATION}

As presented in Romão et al. (2004), the bidimensional equation which will be evaluated in this paper is a parabolic equation, that is, an equation of first order in time and of second order in space. The method used for the discretization in time of equation (1), is a method from the family of $\alpha$ approach, in which the moderated average from the derivated in relation to the time of the dependent variable is approached by two consecutive steps in time by a linear interpolation of the values of the variable for the two steps

$$
\begin{gathered}
\frac{\partial u}{\partial t} \approx \frac{\tilde{u}\}^{s+1}-\{\tilde{u}\}^{s}}{\Delta t^{s+1}}=(1-\alpha) \cdot\{u\}^{s}+(\alpha) \cdot\{u\}^{s+1} \\
\text { for } 0 \leq \alpha \leq 1
\end{gathered}
$$

in which \{\}$^{\mathrm{s}}$ refers to the value of the variable in the step "s" and $\Delta t^{s+1}=t^{s+1}-t^{s}$ is the $(\mathrm{s}+1)$ step in time. In this paper the Crank-Nicolson scheme in which $\alpha=1 / 2$ will be used (Chung, 2002).

\section{SPATIAL DISCRETIZATION}

In this item the spatial discretization of Eq.(1) will be presented by the formulations of Galerkin and the Least Square Finite Element Methods.

\subsection{Galerkin Method}

Initially, the discretization must be made approaching the $u$ function described in Eq. (1) by a function $\hat{u}$, as follows

$$
\tilde{u} \approx \hat{u}^{e}=\sum_{j=1}^{N_{\text {nodes }}} N_{j} \hat{u}_{j}^{e}
$$

and $N_{\text {nodes }}$ is the number of nodes in each element, $N_{j}$ are the interpolation functions and $\hat{u}_{j}^{e}$ are the nodal values of $u$ in the element. By the described approach in Eq. (3) and using Eq. (1), a residual is defined as

$$
\begin{aligned}
& R=\left\{\frac{\psi}{\Delta t} \tilde{u}+\alpha\left[\frac{\partial}{\partial x}\left(k \frac{\partial \tilde{u}}{\partial x}\right)+\frac{\partial}{\partial y}\left(k \frac{\partial \tilde{u}}{\partial y}\right)+A_{x} \frac{\partial \tilde{u}}{\partial x}+\right.\right. \\
& \left.\left.A_{y} \frac{\partial \tilde{u}}{\partial y}+B \tilde{u}\right]\right\}^{s+1}+f+\left\{-\frac{\psi}{\Delta t} \tilde{u}+(1-\alpha) \times\left[\frac{\partial}{\partial x}\left(k \frac{\partial \tilde{u}}{\partial x}\right)+\right.\right. \\
& \left.\left.+\frac{\partial}{\partial y}\left(k \frac{\partial \tilde{u}}{\partial y}\right)+A_{x} \frac{\partial \tilde{u}}{\partial x}+A_{y} \frac{\partial \tilde{u}}{\partial y}+B \tilde{u}\right]\right\}^{s}
\end{aligned}
$$

Now, it is possible to start introducing the approach by the Galerkin Finite Method in which there is the need to define the variational formulation of the generical problem (1), as follows. It must be found $\hat{u}^{e} \in V^{e}, V^{e} \in C^{2}(\Omega)$ in a way that

$$
\int_{\Omega^{e}} R v_{i}^{e} d \Omega=0, \forall v_{i}^{e} \in V^{e}, i=1,2, \ldots, N_{\text {nodes }}
$$

in which $\Omega \subset \mathfrak{R}^{2}$ is a limited and closed domain. Equation (5) will be integrated for any $v_{i}^{e}=N_{i}$, $i=1,2, \ldots, N_{\text {nodes }}$, that is, in the Galerkin Finite Method the weight function is equal to the interpolation function. Using Eq. (3), effecting the proper integrations and some algebra we have the following matricial linear system

$$
[K]\left\{\hat{u}^{s+1, e}\right\}=\{F\}-[H]\left\{\hat{u}^{s, e}\right\}
$$

in which 


$$
\begin{gathered}
K_{i j}=-\int_{\Omega^{e}} \alpha k \frac{\partial N_{i}}{\partial x} \frac{\partial N_{j}}{\partial x} d \Omega-\int_{\Omega^{e}} \alpha k \frac{\partial N_{i}}{\partial y} \frac{\partial N_{j}}{\partial y} d \Omega+ \\
+\int_{\Omega^{e}} \alpha A_{x} N_{i} \frac{\partial N_{j}}{\partial x} d \Omega+\int_{\Omega^{e}} \alpha A_{y} N_{i} \frac{\partial N_{j}}{\partial y} d \Omega+ \\
\quad+\int_{\Omega^{e}}\left(\frac{\psi}{\Delta t}+\alpha B\right) N_{i} N_{j} d \Omega \\
H_{i j}=-\int_{\Omega^{e}}(1-\alpha) k \frac{\partial N_{i}}{\partial x} \frac{\partial N_{j}}{\partial x} d \Omega- \\
-\int_{\Omega^{e}}(1-\alpha) k \frac{\partial N_{i}}{\partial y} \frac{\partial N_{j}}{\partial y} d \Omega+\int_{\Omega^{e}}(1-\alpha) A_{x} N_{i} \frac{\partial N_{j}}{\partial x} d \Omega \\
\quad+\int_{\Omega^{e}}(1-\alpha) A_{y} N_{i} \frac{\partial N_{j}}{\partial y} d \Omega+ \\
\int_{\Omega^{e}}\left(\frac{-\psi}{\Delta t}+(1-\alpha) B\right) N_{i} N_{j} d \Omega \\
F_{i}=-\int_{\Omega^{e}} f N_{i} d \Omega
\end{gathered}
$$

with $i, j=1,2, \ldots$, Nnodes. Further details about the formulation described before can be found in Romão (2004).

\subsection{Least Square Finite Element Method}

The approach by the Least Square Method has as initial characteristic the addition of two extra variables in the problem by the following equations

$$
q_{x}=-k \frac{\partial u}{\partial x}, \quad q_{y}=-k \frac{\partial u}{\partial y}
$$

then the problem is not represented by the partial differentiated equation of second order anymore, but it is a system of three partial differential equations of first order. After replacing the Eqs. (8a-b) in the Eq. (1) and time discretization we have

$$
\begin{aligned}
& \psi \frac{\tilde{u}^{s+1}-\tilde{u}^{s}}{\Delta t}=\alpha\left[\frac{\partial \tilde{q}_{x}}{\partial x}+\frac{\partial \tilde{q}_{y}}{\partial y}-A_{x} \frac{\partial \tilde{u}}{\partial x}-A_{y} \frac{\partial \tilde{u}}{\partial y}-B \tilde{u}\right]^{s+1} \\
& -f+(1-\alpha)\left[\frac{\partial \tilde{q}_{x}}{\partial x}+\frac{\partial \tilde{q}_{y}}{\partial y}-A_{x} \frac{\partial \tilde{u}}{\partial x}-A_{y} \frac{\partial \tilde{u}}{\partial y}-B \tilde{u}\right]^{s}
\end{aligned}
$$

(9a)

$$
\tilde{q}_{x}+k \frac{\partial \tilde{u}}{\partial x}=0, \quad \tilde{q}_{y}+k \frac{\partial \tilde{u}}{\partial y}=0,
$$

Now, we effect the approximation in each element of the functions $\tilde{u}, \tilde{q}_{x}$ e $\tilde{q}_{y}$ by the functions $\hat{u}^{e}, \hat{q}_{x}^{e}$ e $\hat{q}_{y}^{e}$ in the following way:

$$
\begin{array}{lr}
\tilde{u} \cong \hat{u}^{e}=\sum_{i=1}^{\text {Nnode }} N_{i} \hat{u}_{i}^{e} ; & \tilde{q}_{x} \cong \hat{q}_{x}^{e}=\sum_{i=1}^{\text {Nnode }} N_{i} \hat{q}_{x i}^{e} ; \\
\tilde{q}_{y} \cong \hat{q}_{y}^{e}=\sum_{i=1}^{\text {Nnode }} N_{i} \hat{q}_{y_{i}}^{e}, & (10 \mathrm{a}-\mathrm{c})
\end{array}
$$

for any step in time and with Nnode being the number of nodes in each element.

After the spatial approximation be defined, we can write the residual equations of Eqs. (10 a-c) as follows

$$
\begin{aligned}
& R_{1}(x, y)=\left\{\frac{\psi}{\Delta t} \hat{u}+\alpha\left[-\frac{\partial \hat{q}_{x}}{\partial x}-\frac{\partial \hat{q}_{y}}{\partial y}+A_{x} \frac{\partial \hat{u}}{\partial x}+\right.\right. \\
& \left.\left.A_{y} \frac{\partial \hat{u}}{\partial y}+B \hat{u}\right]\right\}^{s+1}+f+\left\{-\frac{\psi}{\Delta t} \hat{u}+(1-\alpha)\left[-\frac{\partial \hat{q}_{x}}{\partial x}-\right.\right. \\
& \left.\left.-\frac{\partial \hat{q}_{y}}{\partial y}+A_{x} \frac{\partial \hat{u}}{\partial x}+A_{y} \frac{\partial \hat{u}}{\partial y}+B \hat{u}\right]\right\}^{s}
\end{aligned}
$$

$$
R_{2}(x, y)=\hat{q}_{x}+k \frac{\partial \hat{u}}{\partial x}, \quad R_{3}(x, y)=\hat{q}_{y}+k \frac{\partial \hat{u}}{\partial y}
$$

The basic idea of the Least Square Finite Element Method is determining $\hat{u}^{e} \in V^{e}$ as minimizer of the functional (Jiang, 1998)

$$
\begin{array}{r}
I\left(R_{1}, R_{2}, R_{3}\right)=\int_{\Omega^{e}} R_{1}^{2}(x, y) d \Omega+\int_{\Omega^{e}} R_{2}^{2}(x, y) d \Omega \\
+\int_{\Omega^{e}} R_{3}^{2}(x, y) d \Omega
\end{array}
$$

that is, the first variation of the functional of $\hat{u}, \hat{q}_{x}$ e $\hat{q}_{y}$ results in

$$
\begin{aligned}
& \int_{\Omega^{e}}\left(\delta R_{1}\right) R_{1} d \Omega+\int_{\Omega^{e}}\left(\delta R_{2}\right) R_{2} d \Omega+ \\
& \quad+\int_{\Omega^{e}}\left(\delta R_{3}\right) R_{3} d \Omega=0
\end{aligned}
$$

Making the appropriate algebrism and necessary integrations, the Eq. (13) generates the following matricial linear system.

$$
\left[\begin{array}{ccc}
A & B & C \\
B^{T} & D & E \\
C^{T} & E^{T} & G
\end{array}\right]\left\{\begin{array}{l}
\left\{\hat{u}^{e, s+1}\right. \\
\hat{q}_{x}^{e, s+1} \\
\left.\hat{q}_{y}^{e, s+1}\right\}
\end{array}\right\}=\left[\begin{array}{c}
F_{1} \\
F_{2} \\
F_{3}
\end{array}\right]-\left[\begin{array}{ccc}
H & I & J \\
M & K & L \\
P & S & O
\end{array}\right]\left\{\begin{array}{l}
\left\{\hat{u}^{e, s}\right. \\
\left\{\hat{q}_{x}^{e, s}\right. \\
\hat{q}_{y}^{e, s}
\end{array}\right\}
$$

in which 


$$
\begin{gathered}
A_{i j}=\int_{\Omega^{e}}\left\{\left[\alpha A_{x} \frac{\partial N_{i}}{\partial x}+\alpha A_{y} \frac{\partial N_{i}}{\partial y}+\left(\frac{\psi}{\Delta t}+\alpha B\right) N_{i}\right] \times\right. \\
{\left[\alpha A_{x} \frac{\partial N_{j}}{\partial x}+\alpha A_{y} \frac{\partial N_{j}}{\partial y}+\left(\frac{\psi}{\Delta t}+\alpha B\right) N_{j}\right]+} \\
\left.+k^{2} \frac{\partial N_{i}}{\partial x} \frac{\partial N_{j}}{\partial x}+k^{2} \frac{\partial N_{i}}{\partial y} \frac{\partial N_{j}}{\partial y}\right\} d \Omega
\end{gathered}
$$

$$
B_{i j}=\int_{\Omega^{e}}\left\{\left[\alpha A_{x} \frac{\partial N_{i}}{\partial x}+\alpha A_{y} \frac{\partial N_{i}}{\partial y}+\left(\frac{\psi}{\Delta t}+\alpha B\right) N_{i}\right] \times\right.
$$$$
\left.\left[-\alpha \frac{\partial N_{j}}{\partial x}\right]+k \frac{\partial N_{i}}{\partial x} N_{j}\right\} d \Omega
$$

$C_{i j}=\int_{\Omega^{e}}\left\{\left[\alpha A_{x} \frac{\partial N_{i}}{\partial x}+\alpha A_{y} \frac{\partial N_{i}}{\partial y}+\left(\frac{\psi}{\Delta t}+\alpha B\right) N_{i}\right] \times\right.$

$$
\left.\left[-\alpha \frac{\partial N_{j}}{\partial y}\right]+k \frac{\partial N_{i}}{\partial y} N_{j}\right\} d \Omega
$$

$D_{i j}=\int_{\Omega^{e}}\left[\alpha \frac{2}{\partial x} \frac{\partial N_{i}}{\partial x}+\frac{\partial N_{j}}{\partial x}+N_{j}\right] d \Omega$

$E_{i j}=\int_{\Omega^{e}} \alpha^{2} \frac{\partial N_{i}}{\partial x} \frac{\partial N_{j}}{\partial y} d \Omega$

(15e)

$$
\begin{aligned}
G_{i j}= & \int_{\Omega^{e}}\left[\alpha \frac{\partial N_{i}}{\partial y} \frac{\partial N_{j}}{\partial y}+N_{i} N_{j}\right] d \Omega \quad(15 f) \\
H_{i j}= & \int_{\Omega^{e}}\left\{\left[\alpha A_{x} \frac{\partial N_{i}}{\partial x}+\alpha A_{y} \frac{\partial N_{i}}{\partial y}+\left(\frac{\psi}{\Delta t}+\alpha B\right) N_{i}\right] \times\right. \\
& \times\left[(1-\alpha) A_{x} \frac{\partial N_{j}}{\partial x}+(1-\alpha) A_{y} \frac{\partial N_{j}}{\partial y}+\left(-\frac{\psi}{\Delta t}+\right.\right. \\
& \left.\left.+(1-\alpha) B) N_{j}\right]\right\} d \Omega \\
I_{i j}=\int_{\Omega^{e}} & {\left[\alpha A_{x} \frac{\partial N_{i}}{\partial x}+\alpha A_{y} \frac{\partial N_{i}}{\partial y}+\left(\frac{\psi}{\Delta t}+\alpha B\right) N_{i}\right] \times } \\
\times & (15 \mathrm{~g})
\end{aligned}
$$

$$
\begin{array}{r}
J_{i j}=\int_{\Omega^{e}}\left[\alpha A_{x} \frac{\partial N_{i}}{\partial x}+\alpha A_{y} \frac{\partial N_{i}}{\partial y}+\left(\frac{\psi}{\Delta t}+\alpha B\right) N_{i}\right] \times \\
\times\left[-(1-\alpha) \frac{\partial N_{j}}{\partial y}\right] d \Omega \\
M_{i j}=\int_{\Omega^{e}}\left[-\alpha \frac{\partial N_{i}}{\partial x}\right] \times\left[(1-\alpha) A_{x} \frac{\partial N_{j}}{\partial x}+(1-\alpha) \times\right. \\
\left.\times A_{y} \frac{\partial N_{j}}{\partial y}+\left(-\frac{\psi}{\Delta t}+(1-\alpha) B\right) N_{j}\right] d \Omega
\end{array}
$$

$$
K_{i j}=\int_{\Omega^{e}}(1-\alpha) \alpha \frac{\partial N_{i}}{\partial x} \frac{\partial N_{j}}{\partial x} d \Omega
$$

$$
L_{i j}=\int_{\Omega^{e}}(1-\alpha) \alpha \frac{\partial N_{i}}{\partial x} \frac{\partial N_{j}}{\partial y} d \Omega
$$

$$
\begin{aligned}
P_{i j}=\int_{\Omega^{e}} & {\left[-\alpha \frac{\partial N_{i}}{\partial y}\right] \times\left[(1-\alpha) A_{x} \frac{\partial N_{j}}{\partial x}+(1-\alpha) \times\right.} \\
& \left.\times A_{y} \frac{\partial N_{j}}{\partial y}+\left(-\frac{\psi}{\Delta t}+(1-\alpha) B\right) \sum_{1 j}\right] d \Omega \\
S_{i j}= & \int_{\Omega^{e}} \alpha(1-\alpha) \frac{\partial N_{i}}{\partial y} \frac{\partial N_{j}}{\partial x} d \Omega
\end{aligned}
$$

$$
O_{i j}=\int_{\Omega^{e}}(1-\alpha) \alpha \frac{\partial N_{i}}{\partial y} \frac{\partial N_{j}}{\partial y} d \Omega
$$

$$
\begin{array}{r}
F_{1_{i}}=\int_{\Omega^{e}}(-f) \times\left[\alpha A_{x} \frac{\partial N_{i}}{\partial x}+\alpha A_{y} \frac{\partial N_{i}}{\partial y}+\right. \\
\left.+\left(\frac{\psi}{\Delta t}+\alpha B\right) N_{i}\right] d \Omega
\end{array}
$$

$$
\begin{aligned}
& F_{2 i}=\int_{\Omega^{e}}(-f) \times\left[-\alpha A_{x} \frac{\partial N_{i}}{\partial x}\right] d \Omega \\
& F_{3 i}=\int_{\Omega^{e}}(-f) \times\left[-\alpha A_{y} \frac{\partial N_{i}}{\partial y}\right] d \Omega
\end{aligned}
$$

with $i, j=1, \ldots$, Nnodes.

\section{NUMERICAL APPLICATIONS}

All the coefficients involved in the matricial systems (6) and (14), respectively from the GFEM and the LSFEM are functions of the space coordinates $x$ and $y$. In this paper the Gaussiam Quadrature ruler (Reddy, 1993) will be used to calculate the integrals found in the matricial system, for that it is necessary to rewrite the integrals in 
terms of a reference coordinates $\xi$ and $\eta$ $(-1 \leq \xi, \eta \leq 1)$, as described in Romão (2004). The interpolations functions in reference coordinates for quadrangular and triangular elements can be found in Dhatt and Touzot (1984).

Right after, two numerical applications will be presented. The first one is about a problem totally diffuse and the second one is about a diffusiveconvective problem with convective dominant terms, with the objective of analyzing the performance of the Galerkin and the Least Square Methods for the mesh previously determined.

Regarding the boundary conditions, the first application presents a case with three boundary conditions of the prescribed function type and one of the prescribed flux ones (isolation) (Figure 1), while the second application of this chapter presents a case with two boundary conditions of the prescribed function type and two of the prescribed flux ones (isolation).

\section{1- Pure Transient Diffusion}

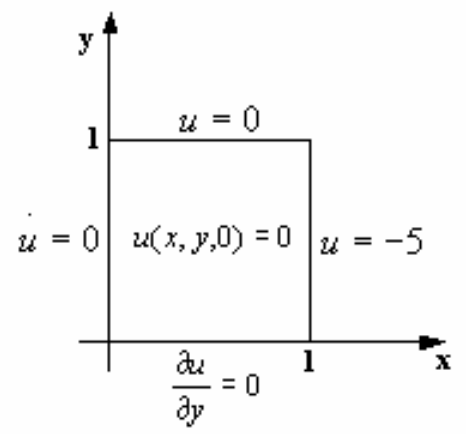

Figure 1. Cartesian Geometry with boundary conditions of the first and second type.

\section{Governing Equation:}

$$
\frac{\partial u(x, y, t)}{\partial t}=\frac{\partial^{2} u(x, y, t)}{\partial x^{2}}+\frac{\partial^{2} u(x, y, t)}{\partial y^{2}}
$$

In this situation, the numerical solution of a completely diffusive case in transient regime is presented for the instant $t=1$. The mesh used in this application is uniform and contains 625 quadrilaterals of 8 nodes, all the elements being of size $h=0,04$. For the time discretization the steps $\Delta t$ $=10^{-2}$ and $\alpha=0,5$ were used. The Figures show the results for the first case analyzed. The values computed are the functions and their first derivatives.

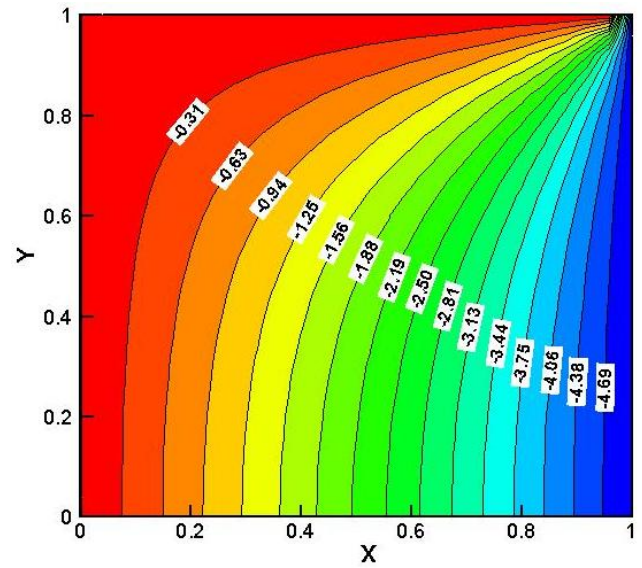

Figure 2. Numerical solutions of $u(x, y, t)$ by GFEM.

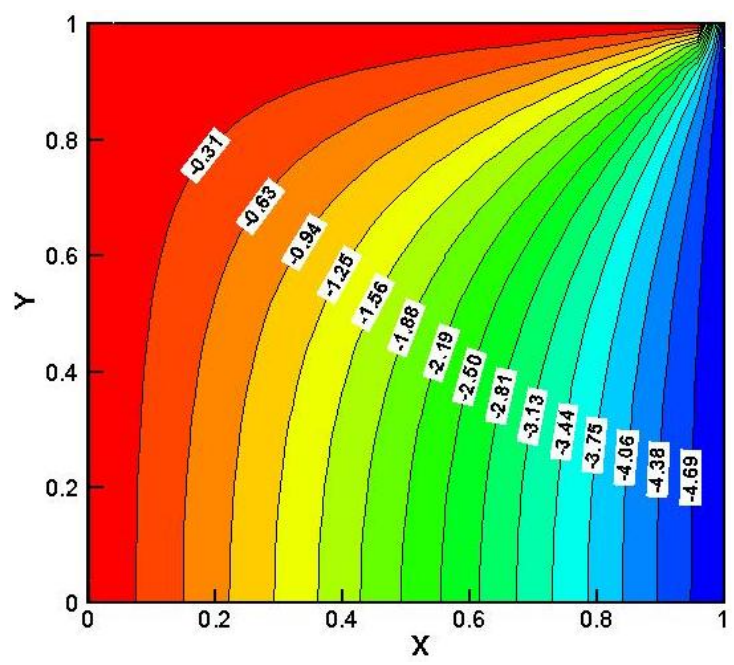

Figure 3. Numerical solutions of $u(x, y, t)$ by LSFEM.

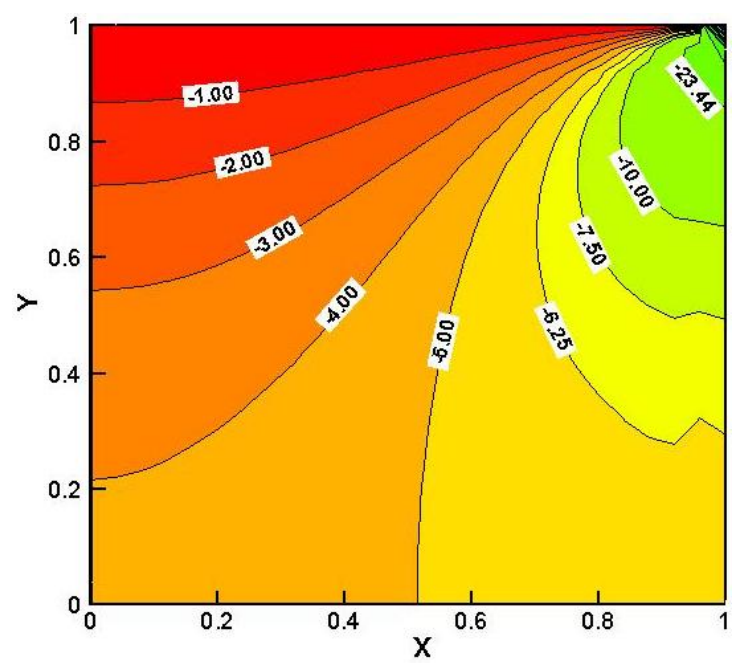

Figure 4. Numerical solutions of $\frac{\partial u}{\partial x}$ by GFEM. 


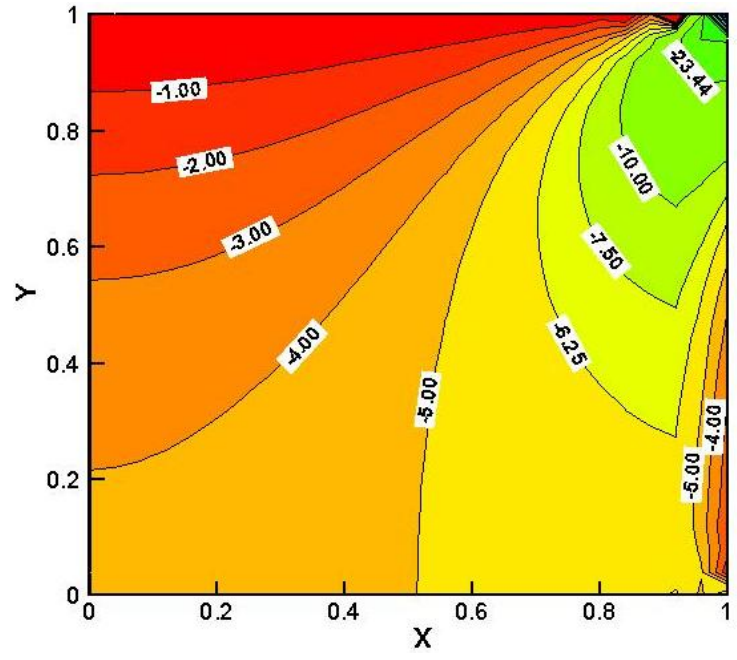

Figure 5. Numerical solutions of $\frac{\partial u}{\partial x}$ by LSFEM.

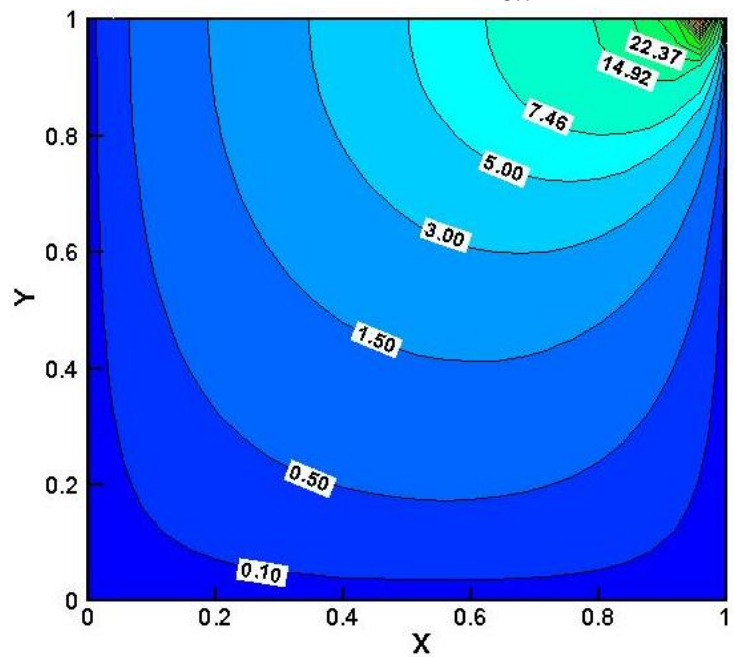

Figure 6. Numerical solutions of the $\frac{\partial u}{\partial y}$ by GFEM.

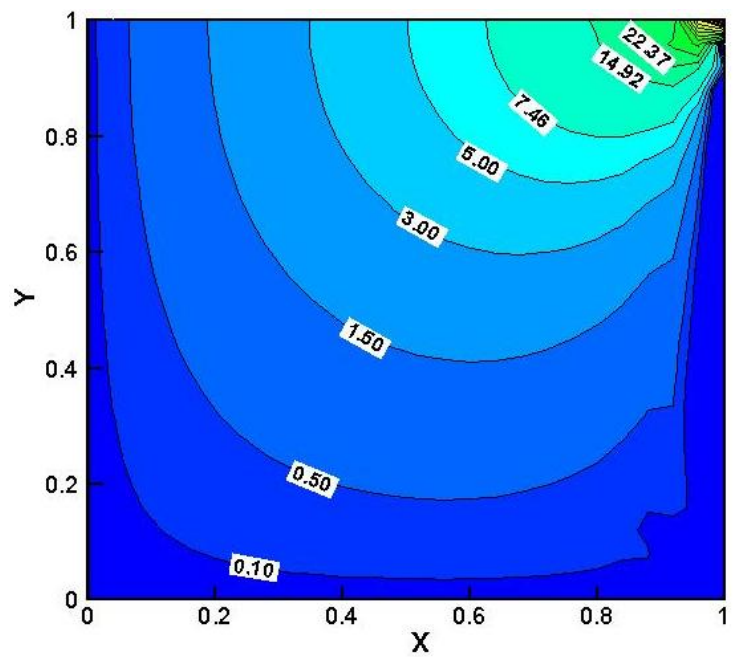

Figure 7. Numerical solutions of the $\frac{\partial u}{\partial y}$ by LSFEM.
Analyzing Figures 2 - 7, we can notice that the GFEM presented good results in all the solutions what did not happen with the LSFEM which presented some numerical oscillations in the solution of $\frac{\partial u}{\partial x}$ and $\frac{\partial u}{\partial y}$ in the proximities of the $x=1$ side. As expected the GFEM presented good results for a purely diffusive problem. The oscillations of the LSFEM may due to the gross mesh.

The initial condition is the prescribed function type according to Fig. 8.

\subsection{Transient Convection-Diffusion}

\section{Governing Equation:}

$$
\frac{\partial u}{\partial t}+10 \frac{\partial u}{\partial x}+10 \frac{\partial u}{\partial y}=\frac{\partial^{2} u}{\partial x^{2}}+\frac{\partial^{2} u}{\partial y^{2}}
$$

with $u=u(x, y, t)$.

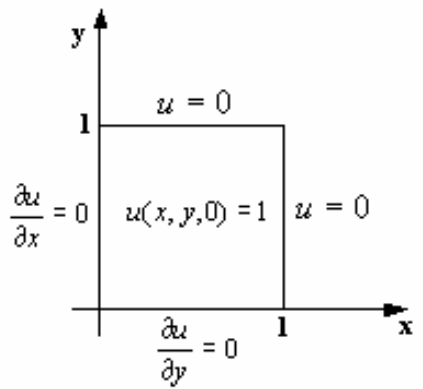

Figure 8. Cartesian Geometry with boundary conditions of the first and second type.

The mesh used in this application contains 1250 triangular elements of six nodes, and all the elements are of identical size $h=0,04 \sqrt{2}$. Figures $9-14$ present numerical solutions for the instant $t=0,5$. In the time discretization, $\Delta t=10^{-2}$ and $\alpha=0,5$ was adopted.

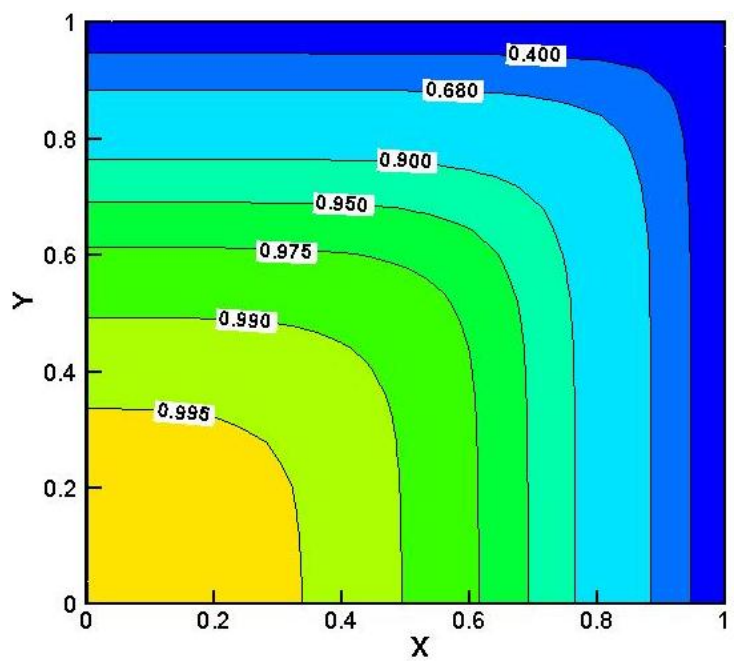

Figure 9. Numerical solution of $u(x, y, t)$ by GFEM. 


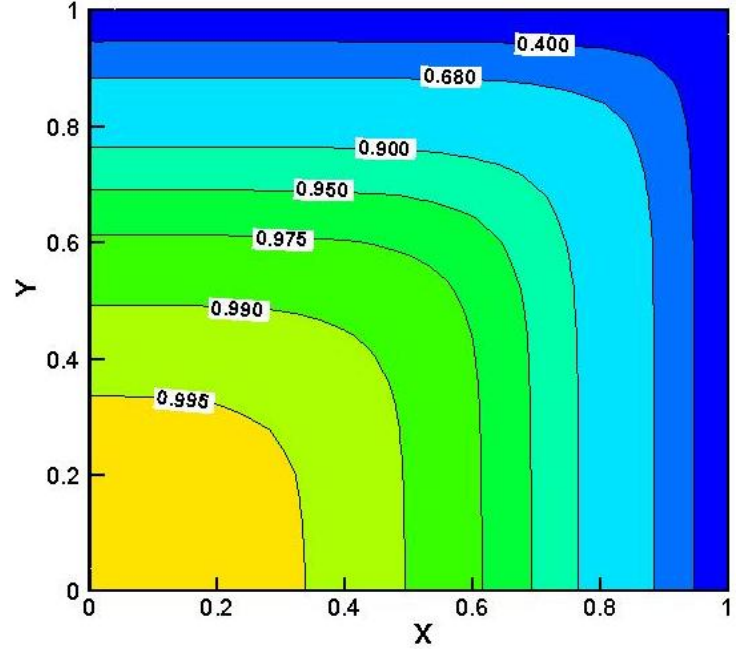

Figure 10. Numerical solution of $u(x, y, t)$ by LSFEM.

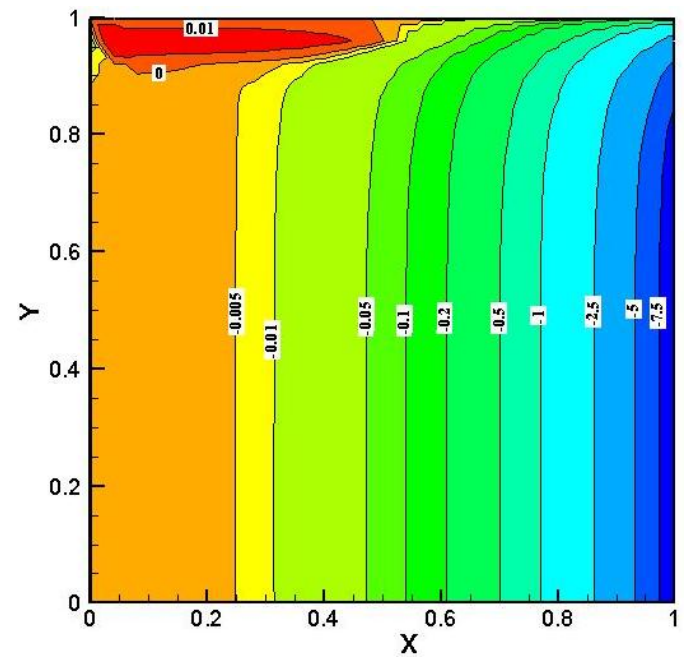

Figure 11. Numerical solutions of $\frac{\partial u}{\partial x}$ by GFEM.

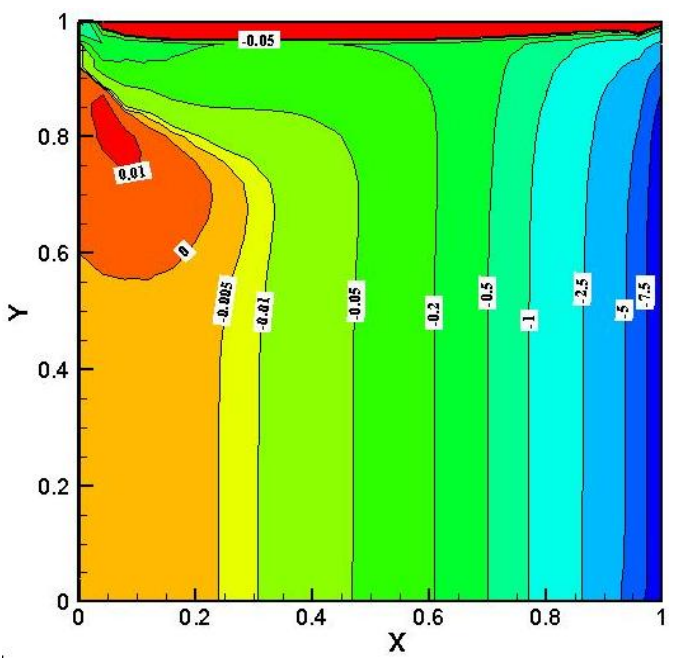

Figure 12. Numerical solutions of $\frac{\partial u}{\partial x}$ by LSFEM.

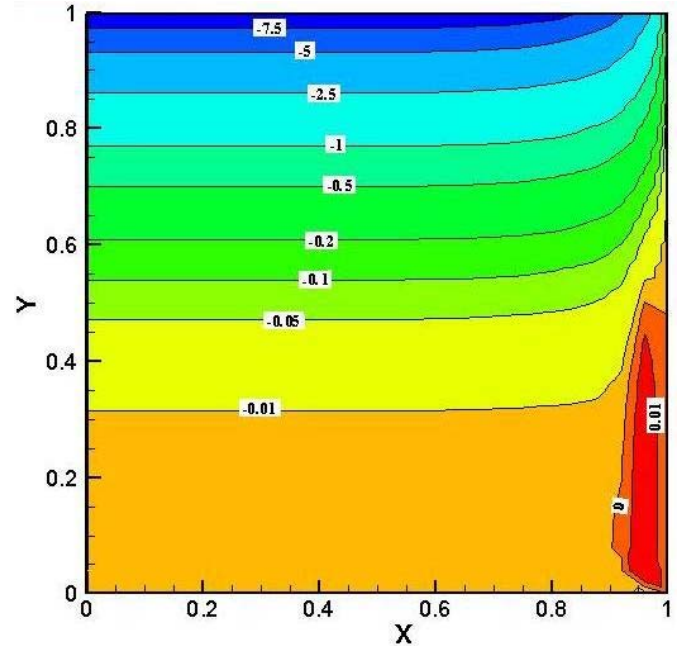

Figure 13. Numerical solutions of $\frac{\partial u}{\partial y}$ by GFEM.

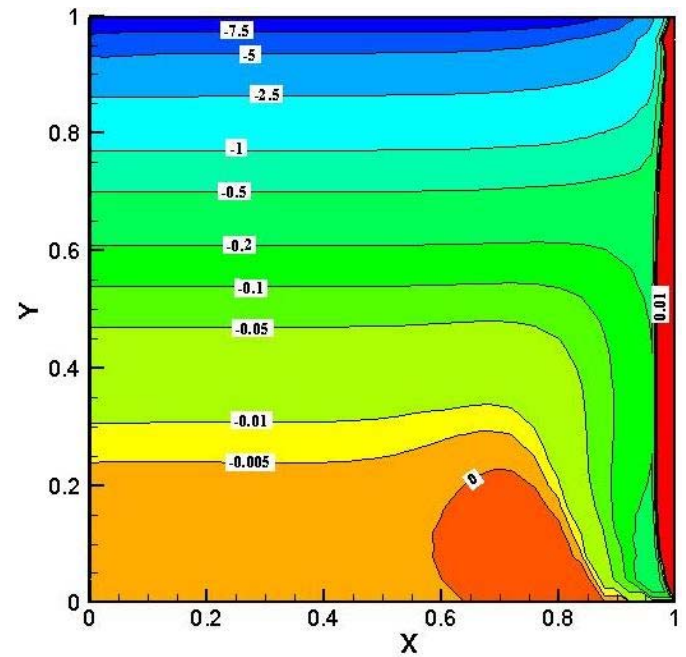

Figure 14. Numerical solutions of $\frac{\partial u}{\partial y}$ by LSFEM.

The conclusion from analyzing the Figures from 9 to 14 is that the GFEM and the LSFEM presented good results in all solutions, but for more convective problems the GFEM may present solutions with oscillations. Some difference appears in the derivative calculation. For the LSFEM the derivative are calculated directly as part of the solution, while for the GFEM the calculation of the derivatives require a post-processing.

\section{CONCLUSIONS}

The LSFEM always results in symmetrical and positive defined algebraic system, which is interesting from the view point of solution methods and revealed to be a good tool in the solution of transient convective-diffusive problems. When the LSFEM was applied to the dominant convective problem proposed, it presented satisfactory results, decreasing the oscillations in the areas next to $\mathrm{x}=1 \mathrm{e}$ 
$y=1$ while in the GFEM the oscillations were more expressive.

\section{ACKNOWLEDGMENTS}

The present work was supported by the National Council of Scientific Development and Technology $\mathrm{CNPq}-$ Brasil, in form of scholarship for the first author.

\section{REFERENCES}

Argyris, J. H., 1963, Recent Advances in Matrix Methods of Structural Analysis, Pergamon Press, Elmsford, New York.

Camprub, N., Colominas, I., Navarrina, F. and Casteleiro, M., 2000, Galerkin, Least-Squares and G.L.S. numerical approaches for convectivediffusive transport problems in engineering, European Congress on Computational Methods in Applied Sciences and Engineering.

Chung, T. J., 2002, Computational Fluid Dynamics, Cambridge University Press.
Clough, R. W., 1960, The Finite Element Method in Plane Stress Analysis, Proceedings of $2^{\text {nd }}$ Conf. on Electronic Computation, American Society of Civil Engineers, Pittsburgh, Penn., pp. 345-378.

Dhatt, G. \& Touzot, G., 1984, The Finite Element Method Displayed, John Wiley \& Sons.

Jiang, Bo-nan, 1998, The Least-Squares Finite Element Method. Theory and Applications in Computational Fluid Dynamics and Electromagnetics, Springer.

Reddy, J. N., 1993, An Introduction to the Finite Element Method, Second Edition, McGrawHill.

Romão, E. C., 2004, Variantes do Método dos Elementos Finitos para Solução de Fenômenos Convectivo-Difusivos Bidimensional, Dissertação de Mestrado, UNESP-FEIS. (in Portuguese)

Romão, E. C. ; Moura, L. F. M. ; Silva, J. B. C., 2008. Analysis of Error in the Solution of the 2-D Diffusion Equation by Finite Element Methods. TEMA. Tendências em Matemática Aplicada e Computacional, Vol. 9, No. 2, pp. 287-298

Turner, M. J., Clough, R. W., Martin, H. C., and Topp, L. P., 1956, Stiffness and Deflection Analysis of Complex Structures, J. Aeron. Sci., Vol. 23, No. 9, pp. 805-823.

Received: August 03, 2008
Revised: September 03, 2008

Accepted: October 03, 2008 